\title{
Technical note: Effect of delayed analysis of cooled lithium-heparinized whole blood on the stability of ionized calcium, ionized magnesium, sodium, potassium, chloride, glucose, and lactate in samples from dairy cows
}

\author{
P. R. Menta, T. A. Batchelder, and R. C. Neves*† \\ Department of Veterinary Sciences, College of Agricultural Sciences and Natural Resources, Texas Tech University, Lubbock 79409
}

\begin{abstract}
The objectives of this study were to describe the stability of bovine whole-blood electrolytes, glucose, and lactate in samples collected in lithium heparin tubes and stored in thermoconductive modules immersed in ice water. A total of 99 Jersey cows (40 first-parity, 18 second-parity, and 41 third-parity or greater cows) from a commercial dairy farm in West Texas were enrolled between June and July 2018. Blood was collected from the jugular vein using a $60-\mathrm{mL}$ polypropylene syringe and equally distributed into 5 spray-dried evacuated lithium heparin tubes. Baseline samples were analyzed within $90 \mathrm{~s}$ of collection using a benchtop blood gas analyzer. The remaining 4 tubes were stored in a thermoconductive, passive-temperature-regulating module inside a cooler with ice water. At $30 \mathrm{~min}$ and 2, 4, and $8 \mathrm{~h}$ post-collection, samples were removed from the temperature-regulating module, gently inverted for 10 $\mathrm{s}$, and analyzed. Repeated-measures models were built to evaluate the effect of time on the stability of ionized $\mathrm{Ca}$ (iCa), ionized $\mathrm{Mg}$ (iMg), $\mathrm{Na}, \mathrm{K}, \mathrm{Cl}$, glucose, and lactate. Most of the analytes investigated remained stable up to $8 \mathrm{~h}$ under ice water storage conditions before analysis, including $\mathrm{iCa}, \mathrm{iMg}, \mathrm{Cl}$, glucose, and lactate. However, $\mathrm{Na}$ and $\mathrm{K}$ were significantly affected by delayed analysis: Na remained stable up to $4 \mathrm{~h}$ post-collection, but $\mathrm{K}$ was not stable starting at $2 \mathrm{~h}$ post-collection. The results of this study are useful in helping future researchers and consultants to recognize acceptable time delays between whole blood collection and processing or analysis for electrolytes, glucose, and lactate.
\end{abstract}

Key words: blood gas analyzer, electrolyte stability, glucose, lactate

\footnotetext{
Received September 30, 2019.

Accepted January 17, 2020.

*Corresponding author: rcneves@purdue.edu

†Current affiliation: Department of Veterinary Clinical Sciences, College of Veterinary Medicine, Purdue University, West Lafayette,
} IN 47907.

\section{Technical Note}

In population-based studies, it is common to collect a large number of blood samples at once, and then transport them to a laboratory or research site for further processing. The time from sample collection to analysis can lead to preanalytical bias; for instance, the environment samples are exposed to during transport to a laboratory (e.g., temperature of the sample and delayed blood separation) can have differential effects on the stability of the analytes and negatively affect study validity (Oddoze et al., 2012). Blood electrolytes, glucose, and lactate are common analytes measured in studies involving dairy cows in the transition period, because energy and mineral balance are commonly disrupted in that phase and associated with negative health outcomes (Chamberlin et al., 2013; Neves et al., 2017; Lopera et al., 2018). Electrolyte imbalances are not uncommon in fresh cow disorders; for instance, cows with left displaced abomasum have hyponatremia, hypokalemia, hypochloremia, and hypocalcemia (Mokhber Dezfouli et al., 2013). Reduced glucose concentrations have been associated with left displaced abomasum (Geishauser et al., 1998), and increased concentrations have been associated with metritis (Bicalho et al., 2017). Lactate is a prognostic indicator of right displaced abomasum (with or without volvulus; Figueiredo et al., 2006).

Samples requiring delayed analysis are often immediately cooled post-collection to decrease cellular metabolic rate (Burnett et al., 1995). Sample cooling is an approach that began as the recommended method for samples collected in glass syringes that required delayed blood gas analyses (CLSI, 2009). For electrolyte results, it was suggested to store cooled samples for up to 30 min post-collection (Burnett et al., 1995). The cooling of blood samples that require delayed analysis is still common practice, and it is generally perceived to be a reasonable approach for maintaining sample stability. To our knowledge, data are lacking to describe the effects of delayed sample analysis for bovine wholeblood electrolytes, glucose, and lactate measurements when stored under refrigeration. 
The objectives of this study were to evaluate the stability of dairy cattle whole-blood electrolytes, glucose, and lactate in samples collected in lithium heparin tubes and stored in thermoconductive modules immersed in ice water. Our primary analyte of interest was ionized $\mathrm{Ca}(\mathbf{i C a})$, because its stability in stored samples remains questionable. According to the International Federation of Clinical Chemistry, iCa should be measured within $1 \mathrm{~h}$ post-collection and must be refrigerated at $4^{\circ} \mathrm{C}$ (Burnett et al., 1995). Other analytes commonly measured in benchtop and point-of-care blood gas analyzers - including ionized $\mathrm{Mg}$ (iMg), Na, $\mathrm{K}, \mathrm{Cl}$, glucose, and lactate - were of secondary interest and are also reported.

All procedures were reviewed and approved by the Texas Tech University Institutional Animal Care and Use Committee (protocol 18035-04). Because the primary analyte of interest was $\mathrm{iCa}$, we built sample size calculations around that analyte and established them using a modified calculation (without replicate samples) of the critical number of test samples from Westgard et al. (1999):

$$
n=\left[Z \frac{\left(C V A^{2}+C V I^{2}\right)^{\frac{1}{2}}}{D}\right]^{2} .
$$

To find at least a $0.5 \%$ change or deviation $(D)$ in iCa at any time point under evaluation with $95 \%$ confidence $(Z)$ and conservatively assuming a $2.5 \%$ analytical imprecision ( $C V A)$ in single measurements (no sample replicates; $C V I$ ), 96 samples were required.

A total of 99 Jersey cows from a dairy farm in West Texas were enrolled over a period of 4 wk between June and July 2018. The sampling population consisted of 75 postpartum cows (0-48 h after calving), 17 cows from the hospital pen, and 7 dry cows. Cows were restrained in a chute and blood was collected from the jugular vein using a $60 \mathrm{~mL}$ polypropylene syringe; any air bubbles in the syringe were removed. Immediately after collection, 5 evacuated tubes $(9 \mathrm{~mL})$ containing spray-dried lithium heparin (Greiner Bio-One, Monroe, NC) were completely filled (i.e., all tubes contained the same amount of blood with no under- or overfilling), and tubes were gently inverted 8 to 10 times to allow for proper anticoagulant mixing. Throughout the study, all tubes used were from the same lot number (B171036T). From each set of samples, 1 was analyzed within $90 \mathrm{~s}$ post-collection (baseline) in a reference blood gas analyzer (Prime Plus VET; Nova Biomedical, Waltham, MA) in a temperature-controlled room $\left(20-25^{\circ} \mathrm{C}\right)$ located $5 \mathrm{~m}$ from the cow chute. The re- maining 4 tubes were stored in a thermoconductive, passive-temperature-regulating module (CoolRack; BioCision, San Rafael, CA) kept inside a cooler (Stanley Adventure Cooler; Stanley PMI, Seattle, WA) containing ice water. Before initiation of the study, we estimated the effect of sample contact time inside the cooled thermoconductive module on decreasing blood temperature using a real-time temperature logger. For 9-mL blood tubes, it takes 15 to $17 \mathrm{~min}$ for the temperature of a whole-blood sample to decrease from that at the time of collection (i.e., body temperature) to $4^{\circ} \mathrm{C}$. Samples are kept between 1 and $4^{\circ} \mathrm{C}$ as long as ice water remains in the cooler, which we determined to be at least $10 \mathrm{~h}$ in our study. The methodology used in the present study was selected to better simulate situations that researchers and dairy consultants face when they perform herd-level studies or in circumstances that require investigations of fresh cow programs; in such instances, a great number of samples are collected at one time for later analysis (i.e., analysis in batches). At $30 \mathrm{~min}$ and 2,4 , and $8 \mathrm{~h}$ post-collection, samples were removed from the temperature-regulating module, gently inverted for $10 \mathrm{~s}$, and analyzed. At all time points, iCa, iMg, $\mathrm{Na}, \mathrm{K}, \mathrm{Cl}$, glucose, and lactate were measured. The analyzer used in the present study uses direct potentiometry to determine $\mathrm{iCa}, \mathrm{iMg}, \mathrm{Na}, \mathrm{K}$, and $\mathrm{Cl}$. Glucose measurement is based on glucose oxidase and lactate measurement is based on lactate oxidase methodologies. We obtained intra- and inter-CV for the analytes studied by measuring a liquid electrolyte quality control level acquired from the manufacturer of the analyzer (lot number: 18239056); we performed intra-CV using 10 consecutive measurements of the quality control, and we obtained inter-CV from daily quality control measurements across $10 \mathrm{~d}$. Intra- and inter-CV, respectively, were as follows: 1.94 and $0.38 \%$ for iCa; 6.93 and $5.61 \%$ for iMg; 0.56 and $0.61 \%$ for $\mathrm{Na} ; 1.49$ and $1.26 \%$ for K; 0.32 and $0.71 \%$ for $\mathrm{Cl} ; 1.46$ and $1.11 \%$ for glucose; and 4.08 and $2.16 \%$ for lactate. The blood samples from each cow were distributed into 5 different tubes to better maintain the anaerobic conditions of the specimen during the post-collection analysis intervals (i.e., removal of blood using a needle attached to a syringe from a single tube, or simply uncapping samples for stability studies involving iCa and $\mathrm{iMg}$ measurements, would not have allowed us to maintain the sample in an anaerobic environment). In addition, rapid cooling of the samples should be able to better preserve $\mathrm{iCa}$ and $\mathrm{iMg}$ because of a reduction in cellular metabolic rate (i.e., prevention of excessive lactate formation with consequent sample acidification and $\mathrm{Ca}$ and $\mathrm{Mg}$ protein-bound dissociation). Finally, maintenance of the same ratio of blood to lithium heparin across samples prevented a heparin dilution effect. 
We avoided the use of sodium heparin tubes because of our interest in Na stability.

Descriptive statistics and data modeling were developed in SAS (version 9.4; SAS Institute Inc., Cary, $\mathrm{NC}$ ). Linear mixed models were built to evaluate the effect of time (discrete variable) on the stability of each analyte of interest using the MIXED procedure, while accounting for the repeated effect of time; cow ID was accounted for as a random effect. Covariance structures (variance components, first-order autoregressive, compound symmetry, Toeplitz, and Toeplitz heterogeneous) were tested in the final model, and the one yielding the lowest model Akaike information criterion was chosen. In all models, first-order autoregressive was the covariance structure chosen. Influence diagnostics were assessed by Pearson and Studentized residuals; no outliers were observed. If the time variable had a significant effect in the model $(P \leq 0.05)$, multiple comparisons were corrected using Dunnett's method (i.e., comparisons against the baseline concentration).

Median concentration and range (minimum and maximum) in millimoles per liter for baseline iCa, iMg, Na, $\mathrm{K}, \mathrm{Cl}$, glucose, and lactate for the sampling population were as follows: 1.21 (0.72-1.46); 0.69 (0.44-1.24); 141.8 (129.9-147.7); 4.14 (3.30-5.08); 106.4 (98.4-113.0); $4.4(2.8-8.1)$; and $2.0(0.5-5.6)$. Least squares means differences for all analytes measured compared to the baseline value are presented in Table 1 . We found no effect of time on the concentration of iCa $(P=0.99)$. Considerable efforts have been devoted to describing the preanalytical effects that influence iCa measurements in humans; for instance, recommendations rely on avoiding sample $\mathrm{pH}$ changes by preventing $\mathrm{CO}_{2}$ losses and minimizing lactic acid formation (Burnett et al., 1995). In our study, we found an effect of storage time on sample $\mathrm{pH}$ (data not shown) although it was not enough to alter iCa concentrations. The fact that our samples were kept capped and chilled most likely prevented greater disturbances in sample $\mathrm{pH}$ and $\mathrm{iCa}$ concentrations. Szenci et al. (1991) evaluated the stability of iCa in 12 bovine venous blood samples collected in Ca-titrated heparin solution; changes were never greater than $1.2 \%$ for up to $48 \mathrm{~h}$ post-collection. The binding of $\mathrm{Ca}$ ions to heparin salts was a likely preanalytical bias in our study. In human samples, blood collected in lithium heparin tubes had iCa concentrations of $-0.08 \mathrm{mmol} / \mathrm{L}$ on average, compared with samples collected in balanced-heparin tubes (Toffaletti et al., 1991). To overcome the binding of Ca to heparin, Catitrated syringes were manufactured and are commonly adopted in human arterial sampling. Titration is generally set at $1.25 \mathrm{mmol} / \mathrm{L}$ and prevent any measurement bias in samples containing normal iCa concentrations (Toffaletti et al., 1991). However, collection of samples

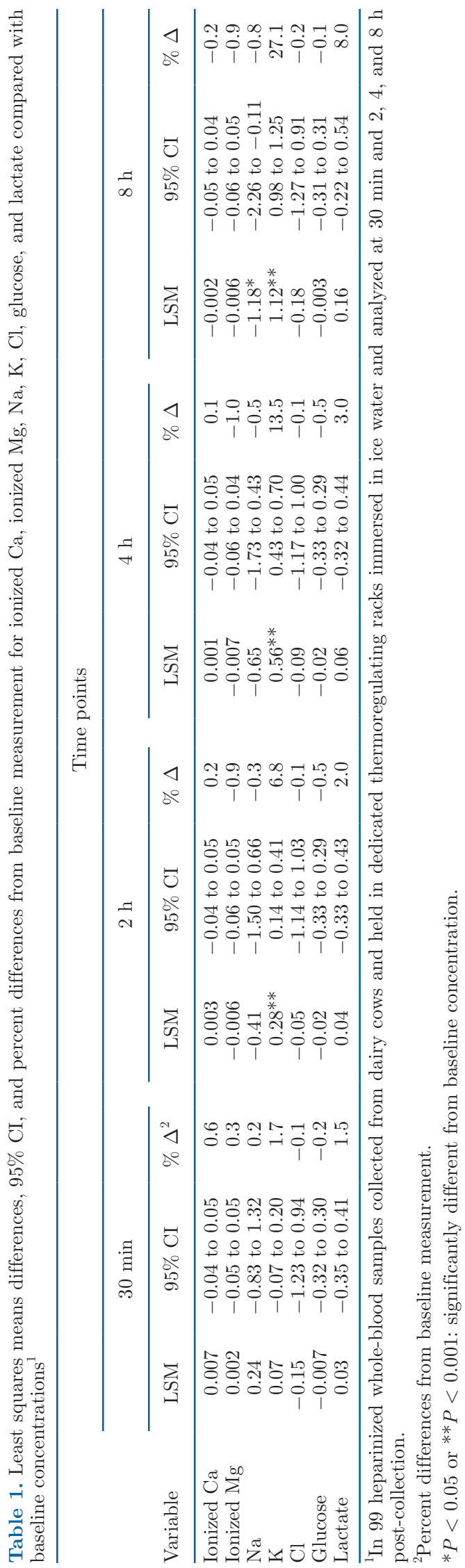


Table 2. Distribution of ionized Ca (iCa) concentration change compared with baseline values ${ }^{1}$

\begin{tabular}{lcccc}
\hline & \multicolumn{4}{c}{ Time points } \\
\cline { 2 - 5 } $\begin{array}{l}\text { Change in baseline iCa } \\
\text { concentration, mmol/L }\end{array}$ & $\Delta 30$ min to $0 \mathrm{~h}$ & $\Delta 2$ to $0 \mathrm{~h}$ & $\Delta 4$ to $0 \mathrm{~h}$ & $\Delta 8$ to $0 \mathrm{~h}$ \\
\hline-0.03 & 0 & 0 & 1 & 3 \\
-0.02 & 0 & 1 & 2 & 4 \\
-0.01 & 6 & 16 & 26 & 32 \\
0 & 37 & 31 & 35 & 37 \\
0.01 & 40 & 8 & 8 & 1 \\
0.02 & 15 & 0 & 0 & 0 \\
\hline .03 & 1 & & 8 & 22 \\
\hline
\end{tabular}

${ }^{1}$ In 99 heparinized whole-blood samples collected from dairy cows and held in dedicated thermoregulating racks immersed in ice water and analyzed at $30 \mathrm{~min}$ and 2,4 , and $8 \mathrm{~h}$ post-collection.

from early-postpartum cows may not be ideal, because a proportion of animals will have iCa lower than normal, leading to a false increase in analyte concentration. This preanalytical bias has been documented in hypocalcemic human samples and requires caution (Toffaletti et al., 1991). In bovine samples, temperature reduction can greatly reduce changes in iCa (Szenci et al., 1994). We found that $\geq 83 \%$ of the samples maintained iCa concentrations within $\pm 0.01 \mathrm{mmol} / \mathrm{L}$ compared with baseline values, and that changes were never greater than $\pm 0.03 \mathrm{mmol} / \mathrm{L}$ for up to $8 \mathrm{~h}$ post-collection (Table $2)$. Therefore, collection of blood in completely filled lithium heparin tubes and appropriate cooling $\left(1-4^{\circ} \mathrm{C}\right)$ of samples immediately after collection constituted a suitable method for preserving iCa diagnostic value for up to $8 \mathrm{~h}$ post-collection. However, the most suitable sample for iCa still remains testing of fresh anticoagulated blood by direct potentiometry, because this will be more closely correlated with in vivo concentrations (Toffaletti et al., 1991; CLSI, 2009).

We found no effect of time on iMg, Cl, glucose $(P$ $=0.99$ in all models $)$, or lactate $(P=0.89)$. We are unaware of studies that specifically evaluated the stability of cooled bovine whole-blood samples for iMg, $\mathrm{Cl}$, glucose, and lactate. No significant differences were observed for up to $6 \mathrm{~h}$ post-collection in a study that evaluated the stability of $\mathrm{iMg}$ in human whole blood (Greenway et al., 1996). Similarly, iMg in feline serum samples were stable for up to $6 \mathrm{~h}$ post-collection (Gilroy et al., 2005). In human samples, $\mathrm{Cl}$ is stable when stored at $4^{\circ} \mathrm{C}$ (Boyanton and Blick, 2002; Oddoze et al., 2012), and our study supports stability for up to $8 \mathrm{~h}$ post-collection in bovine samples.

Delayed glucose measurements are not commonly conducted in whole-blood samples collected in tubes that do not contain antiglycolytic preservatives. The rate of sample glycolysis is known to be temperaturedependent, and cooling is a generally accepted method for samples that will be analyzed within 30 min postcollection (Sacks et al., 2011). However, we are unaware of studies that have evaluated the single effect of sample cooling without antiglycolytic(s) in preserving glucose in bovine whole blood. In humans, immediate cooling of whole-blood samples was able to preserve glucose up to $4 \mathrm{~h}$ post-collection without clinically significant changes (Lin et al., 1976). Prevention of glycolysis has been demonstrated to preserve lactate stability in human samples (Astles et al., 1994). In our study, immediate cooling of the samples meaningfully reduced cell metabolism, because glucose and lactate concentrations remained stable for up to $8 \mathrm{~h}$ post-collection.

It is unknown if (or how) the thermoconductive modules themselves contributed to sample stability in this study. The modules prevented blood tubes from having direct contact with the ice water and a more uniform temperature stabilization; better temperature stabilization is thought to better preserve the analytical quality of the sample and is advantageous to researchers performing population-based studies that require the collection of multiple samples in a short time interval. In addition, by avoiding direct immersion of samples in ice water, sample identification could be better conserved, an advantage for samples requiring transport time.

We found an effect of time on concentrations of $\mathrm{Na}$ $(P=0.01)$ and $\mathrm{K}(P<0.001)$. Samples analyzed at $8 \mathrm{~h}$ post-collection showed an average $\mathrm{Na}$ decrease of $1.18 \mathrm{mmol} / \mathrm{L}$ compared with baseline values; however, cooled samples remained stable until $4 \mathrm{~h}$ after collection. However, despite this significant decrease in $\mathrm{Na}$ concentration $8 \mathrm{~h}$ post-collection, the change remained within analytical quality requirements $( \pm 4 \mathrm{mmol} / \mathrm{L})$ and, therefore, can be considered of low clinical relevance (CLSI, 2000). For K, samples analyzed at 2, 4, and $8 \mathrm{~h}$ post-collection showed significant and progressive increases in concentrations compared with baseline values. Samples analyzed up to $2 \mathrm{~h}$ after collection remained within analytical imprecision limits $( \pm 0.5$ $\mathrm{mmol} / \mathrm{L}$ ), but storage for 4 and $8 \mathrm{~h}$ post-collection led to clinically meaningful changes. The instability of $\mathrm{Na}$ and $\mathrm{K}$ in cooled whole-blood samples is known to be 
related to the inhibition of Na-K-ATPase (Oddoze et al., 2012; Asirvatham et al., 2013).

We investigated the stability of lithium-heparinized blood electrolytes, glucose, and lactate in cooled bovine whole-blood samples. Samples analyzed for iCa, $\mathrm{iMg}, \mathrm{Cl}$, glucose, and lactate were stable up to $8 \mathrm{~h}$ post-collection. Importantly, iCa remained of diagnostic value in cooled whole blood. However, $\mathrm{Na}$ and $\mathrm{K}$ measurements in whole blood were unreliable during prolonged cold storage.

\section{ACKNOWLEDGMENTS}

We thank the participating herd and employees for their cooperation during the study. The authors have stated no conflicts of interest.

\section{REFERENCES}

Asirvatham, J. R., V. Moses, and L. Bjornson. 2013. Errors in potassium measurement: A laboratory perspective for the clinician. N. Am. J. Med. Sci. 5:255-259. https://doi.org/10.4103/1947-2714 .110426 .

Astles, R., C. P. Williams, and F. Sedor. 1994. Stability of plasma lactate in vitro in the presence of antiglycolytic agents. Clin. Chem. 40:1327-1330. https://doi.org/10.1093/clinchem/40.7.1327.

Bicalho, M. L., E. C. Marques, R. O. Gilbert, and R. C. Bicalho. 2017. The association of plasma glucose, BHBA, and NEFA with postpartum uterine diseases, fertility, and milk production of Holstein dairy cows. Theriogenology 88:270-282. https://doi.org/10.1016/j .theriogenology.2016.09.036.

Boyanton, B. L. Jr., and K. E. Blick. 2002. Stability studies of twentyfour analytes in human plasma and serum. Clin. Chem. 48:22422247. https://doi.org/10.1093/clinchem/48.12.2242.

Burnett, R. W., A. K. Covington, N. Fogh-Andersen, W. R. Külpmann, A. H. Maas, O. Müller-Plathe, and O. Siggaard-Andersen. 1995. International Federation of Clinical Chemistry (IFCC), Committee on $\mathrm{pH}$, Blood Gases and Electrolytes: Approved IFCC recommendation on definitions of quantities and conventions related to blood gases and pH. Eur. J. Clin. Chem. Clin. Biochem. 33:399-404.

Chamberlin, W. G., J. R. Middleton, J. N. Spain, G. C. Johnson, M. R. Ellersieck, and P. Pithua. 2013. Subclinical hypocalcemia, plasma biochemical parameters, lipid metabolism, postpartum disease, and fertility in postparturient dairy cows. J. Dairy Sci. 96:7001-7013. https://doi.org/10.3168/jds.2013-6901.

CLSI (Clinical and Laboratory Standards Institute). 2000. C29-A2 Standardization of Sodium and Potassium Ion-Selective Electrode Systems to the Flame Photometric Reference Method. 2nd ed. CLSI, Wayne, PA.

CLSI (Clinical and Laboratory Standards Institute). 2009. C31-A2. Ionized Calcium Determinations: Precollection Variables, Specimen Choice, Collection, and Handling. 2nd ed. CLSI, Wayne, PA.

Figueiredo, M. D., D. V. Nydam, G. A. Perkins, H. M. Mitchell, and T. J. Divers. 2006. Prognostic value of plasma L-lactate concentra- tion measured cow-side with a portable clinical analyzer in Holstein dairy cattle with abomasal disorders. J. Vet. Intern. Med. 20:1463-1470. https://doi.org/10.1111/j.1939-1676.2006.tb00767 $\mathrm{x}$.

Geishauser, T., K. Leslie, T. Duffield, D. Sandals, and V. Edge. 1998. The association between selected metabolic parameters and left abomasal displacement in dairy cows. J Vet Med 45:499-511.

Gilroy, C. V., S. A. Burton, B. S. Horney, and A. L. MacKenzie. 2005. Validation of the Nova CRT8 for the measurement of ionized magnesium in feline serum. Vet. Clin. Pathol. 34:124-131. https://doi .org/10.1111/j.1939-165X.2005.tb00024.x.

Greenway, D. C., J. T. Hindmarsh, J. Wang, J. A. Khodadeen, and P. C. Hebert. 1996. Reference interval for whole blood ionized magnesium in a healthy population and the stability of ionized magnesium under varied laboratory conditions. Clin. Biochem. 29:515-520. https://doi.org/10.1016/S0009-9120(96)00091-4.

Lin, Y. L., C. H. Smith, and D. N. Dietzler. 1976. Stabilization of blood glucose by cooling with ice: An effective procedure for preservation of samples from adults and newborns. Clin. Chem. 22:2031-2033. https://doi.org/10.1093/clinchem/22.12.2031.

Lopera, C., R. Zimpel, A. Vieira-Neto, F. R. Lopes, W. Ortiz, M. Poindexter, B. N. Faria, M. L. Gambarini, E. Block, C. D. Nelson, and J. E. P. Santos. 2018. Effects of level of dietary cation-anion difference and duration of prepartum feeding on performance and metabolism of dairy cows. J. Dairy Sci. 101:7907-7929. https://doi .org/10.3168/jds.2018-14580.

Mokhber Dezfouli, M., Z. Eftekhari, S. Sadeghian, A. Bahounar, and M. Jeloudari. 2013. Evaluation of hematological and biochemical profiles in dairy cows with left displacement of the abomasum. Comp. Clin. Path. 22:175-179. https://doi.org/10.1007/s00580 $-011-1382-5$.

Neves, R. C., B. M. Leno, T. Stokol, T. R. Overton, and J. A. A. McArt. 2017. Risk factors associated with postpartum subclinical hypocalcemia in dairy cows. J. Dairy Sci. 100:3796-3804. https:// doi.org/10.3168/jds.2016-11970.

Oddoze, C., E. Lombard, and H. Portugal. 2012. Stability study of 81 analytes in human whole blood, in serum and in plasma. Clin. Biochem. 45:464-469. https://doi.org/10.1016/j.clinbiochem.2012 .01 .012 .

Sacks, D. B., M. Arnold, G. L. Bakris, D. E. Bruns, A. R. Horvath, M. S. Kirkman, A. Lernmark, B. E. Metzger, and D. M. Nathan. 2011. Guidelines and recommendations for laboratory analysis in the diagnosis and management of diabetes mellitus. Diabetes Care 34:e61-e99. https://doi.org/10.2337/dc11-9998.

Szenci, O., E. Brydl, and C. A. Bajcsy. 1991. Effect of storage on measurement of ionized calcium and acid-base variables in equine, bovine, ovine, and canine venous blood. J. Am. Vet. Med. Assoc. 199:1167-1169.

Szenci, O., F. Nemeth, Z. Stollar, and E. Brydl. 1994. Effects of storage time and temperature on ionized calcium concentration in equine blood, plasma, and serum. J. Am. Vet. Med. Assoc. 204:1224-1226.

Toffaletti, J., P. Ernst, P. Hunt, and B. Abrams. 1991. Dry electrolyte-balanced heparinized syringes evaluated for determining ionized calcium and other electrolytes in whole blood. Clin. Chem. 37:1730-1733. https://doi.org/10.1093/clinchem/37.10.1730.

Westgard, J. O., P. L. Barry, E. F. Quam, and S. S. Ehrmeyer. 1999. Basic Method Validation: Training in Analytical Quality Management for Healthcare Laboratories. Westgard Quality Corporation, Madison, WI 